\title{
Régime alimentaire de Tilapia, Oreochromis niloticus, du Lac Muhazi (Rwanda)
}

\author{
R. Mukankomeje ${ }^{1}$ \\ F. Laviolette 2 \\ J.-P. Descyl*
}

Mots clés : Tilapia, Oreochromis niloticus, régime alimentaire, Cyanobactéries, phytoplancton, Lac Muhazi, Rwanda.

Le régime-alimentaire de Tilapia, Oreochromis niloticus, principale espèce exploitée commercialement au Lac Muhazi, a été étudié de décembre 1989 à septembre 1990, en fonction des saisons, des stations et de la taille des individus capturés dans les filets de pêche. Tous les indices considérés montrent que $O$. niloticus du Lac Muhazi consomme bien le phytoplancton et que les Cyanobactéries sont les plus représentées dans les contenus stomacaux comme c'est le cas dans le lac. Pour les 118 contenus stomacaux analysés, l'indice d'occurence arrive à $100 \%$ pour certaines espèces de Cyanobactéries (Merismopedia tenuissima). Pour ce groupe dominant, l'indice d'abondance est en moyenne de 64,3 \%, l'indice volumétrique de $83 \%$ et l'indice de dominance de Lauzanne de 58,1. Deux espèces, Microcystis aeruginosa et Ceratium hirundinella représentent à elles seules plus de $90 \%$ de la biomasse contenue dans les estomacs des poissons dont la taille est supérieure à $90 \mathrm{~mm}$ quelles que soient la station et la saison de prélèvement. Pour la période d'étude, nous avons constaté que $O$. niloticus a une activité essentiellement diurne (il se nourrit entre $6 \mathrm{~h}$ et $21 \mathrm{~h} 30$ ), avec une ration journalière élevée $\left(26,5 \mathrm{~g}^{-1} \mathrm{j}^{-1}\right)$. Ceci explique sans doute la bonne croissance de cette espèce dans les milieux où le phytoplancton est abondant. Cependant, au Lac Muhazi, la population d'O. niloticus n'atteint qu'une biomasse relativement faible et ne consomme qu'une faible fraction de la production primaire nette $(6 \%)$.

\section{Diet of the Nile Tilapia, Oreochromis niloticus, in lake Muhazi (Rwanda)}

Keywords : Tilapia, Oreochromis niloticus, diet, Cyanobacteria, phytoplankton, Muhazi lake, Rwanda.

The diet of Oreochromis niloticus, the mainly exploited species in Lake Muhazi, was studied from December 1989 to September 1990. The diet was investigated in terms of sites, seasons and size of the specimens caught. O. niloticus feeds on phytoplankton, and Cyanobacteria make up an important fraction in the stomach contents analyzed. In the 118 stomach contents, belonging to fishes larger than $90 \mathrm{~mm}$, the index of occurrence reaches $100 \%$ for some Cyanobacteria (Merismopedia tenuissima). For this dominant group, the mean abundance index is $64,3 \%$, the mean volumetric index is $83 \%$ and the dominance index of Lauzanne is 58,1. Two species, Microcystis aeruginosa and Ceratium hirundinella represent more than $90 \%$ of the biomass in the stomach contents analyzed from the two sites and in all seasons. For the period of study, we established that $O$. niloticus feeds only during the day (from $6 \mathrm{~h}$ to $21 \mathrm{~h} \mathrm{30}$ ) and

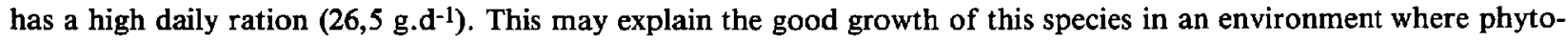
plankton is abundant. However, the population of $O$. niloticus of Lake Muhazi has a relatively small biomass and feeds on a small fraction of the phytoplankton primary production $(6 \%)$.

1. Unité d'Ecologie des Eaux Douces, Facultés Universitaires Notre Dame de'la Paix, Rue de Bruxelles, 61, B-5000 Namur, Belgique.

2. Faculté des Sciences Agronomiques, Unité des Eaux et Forêts, Université Catholique de Louvain, Belgique.

* Auteur pour la correspondance. 


\section{Introduction}

Oreochromis niloticus (Linné, 1758), est l'une des espèces de poissons introduites au Lac Muhazi (Rwanda) vers les années 1935, en provenance du Lac Edouard (De Vos et al. 1990). Bien qu'il consomme ce qu'il trouve dans le milieu, il est essentiellement phytoplanctonophage à l'âge adulte dans la plupart des eaux tropicales (Fish 1955, LoweMcConnel 1958, Blache 1964, Fryer \& Iles 1972, Moriarty \& Moriarty 1973, Bunep 1988, Beveridge et al. 1989, Getachew \& Fernando 1989). Des observations faites ailleurs montrent que $O$. niloticus a une activité diurne (Moriarty \& Moriarty 1973, Dewan \& Shaha 1979, Getachew 1989, Palomarès 1991). Son régime alimentaire varie suivant l'âge et le milieu.

Cette introduction au Lac Muhazi a été considérée comme un succès. Vers 1952 , la production halieutique du lac, à base des tilapias, était estimée à 350 tonnes de poissons par an soit $100 \mathrm{~kg} \cdot \mathrm{ha}^{-1} \cdot$ an $^{-1}$ (Damas 1953). Cependant, la production a chuté par la suite pour atteindre $30 \mathrm{~T}^{\mathrm{an}}{ }^{-1}$ soit 9 kg.ha-1 an $^{-1}$ en 1985 (Plisnier 1990). Des études ont alors été entreprises pour identifier les causes de cette réduction de productivité. Une des hypothèses envisagées est en partie basée sur la comparaison des données de Damas (1953) et des études limnologiques récentes qui semblent indiquer que le lac a subi de fortes modifications au cours des quelque quarante dernières années. On remarque notamment une réduction de la transparence de l'eau et une productivité primaire apparemment faible, si l'on se base sur la biomasse phytoplanctonique (33,7 $\mathrm{mg}$ Chla.m-3 au Lac Muhazi) comparée à d'autres lacs proches $(70 \mathrm{mg}$ Chla.m-3 au Lac Ihema), à productivité piscicole élevée (Plisnier 1990).

Dans ce contexte, le premier objectif du présent travail fut l'évaluation de la production primaire phytoplanctonique du lac, qui est en moyenne de $7,75 \mathrm{~g} \mathrm{O}_{2} \cdot \mathrm{m}^{-2} \cdot \mathrm{j}^{-1}$ (production brute). Cette production situe le lac Muhazi parmi les lacs africains à productivité moyenne (Mukankomeje et al. 1993). De nombreux auteurs (Smith \& Swingleh 1938, Ryder 1965, Sreenivisan 1972, Melack 1976, Oglesby 1977 , McConnel et al. 1977, Liang et al. 1981, Olah et al. 1986, Naca 1989, Downing et al. 1990) ont proposé des relations entre la production primaire et la production piscicole en milieux naturels et dans les piscicultures. L'application de ces relations au Lac Muhazi permet d'estimer la production piscicole exploitable à $65 \mathrm{~kg}$. ha-1. an-1 (Mukankomeje et al. 1993) contre $20 \mathrm{~kg}$.ha-1 an $^{-1}$ exploités actuellement (Plisnier 1990).

La présente contribution est consacrée à l'analyse détaillée du régime alimentaire d' $O$. niloticus, afin de vérifier que cette espèce consomme bien le phytoplancton. Le régime alimentaire a été envisagé en fonction des saisons, des stations de pêche et de la taille des individus capturés. La ration journalière a été aussi évaluée, ce qui a notamment permis de déterminer la fraction de la production phytoplanctonique consommée par $O$. niloticus.

\section{Description du milieu}

Le Lac Muhazi est un lac naturel de vallée barrée, situé au sud de l'équateur, entre $1^{\circ} 49^{\prime}$ et $1^{\circ} 54^{\prime}$ de latitude sud et entre $30^{\circ} 18^{\prime}$ et $30^{\circ} 30^{\prime}$ de longitude est (Fig. $1, n^{\circ} 3$ ). Le niveau du lac est à $1450 \mathrm{~m}$ d'altitude. Il a une superficie de $34,6 \mathrm{~km}^{2}$, une longueur de $37 \mathrm{~km}$ et une largeur moyenne de $0,6 \mathrm{~km}$. Sa profondeur moyenne est de $7,6 \mathrm{~m}$, avec un maximum $(13 \mathrm{~m})$ à l'est et un minimum $(5 \mathrm{~m})$ à l'ouest, vers l'exutoire. Il possède de nombreux bras qui se prolongent en marécages avec des macrophytes (Chara spp., Potamogeton spp.).

Les précipitations annuelles sont en moyenne de 1100 mm (1988-1990). Les géographes (Sirven et al. 1974) subdivisent l'année rwandaise en quatre saisons : une petite saison sèche qui va de décembre à février ; une grande saison des pluies qui commence en mars et finit en mai (cette dernière peut totaliser à elle seule plus de la moitié des précipitations annuelles) ; une grande saison sèche qui débute en juin et se termine normalement en août et enfin, une petite saison des pluies qui a lieu de la miseptembre à fin novembre.

La température moyenne de l'eau de surface est de $24^{\circ} \mathrm{C}$; la différence entre la température de surface et celle du fond est généralement faible $\left(2^{\circ} \mathrm{C}\right)$. Ces faibles écarts se traduisent néanmoins par un gradient de densité suffisant pour maintenir une stratification par temps calme. Cette stratification est cependant assez labile, surtout en fin de nuit, des vents matinaux entraînant souvent un mélange de la masse d'eau. Ces mélanges, étroitement dépendants de la profondeur, sont plus fréquents dans la 


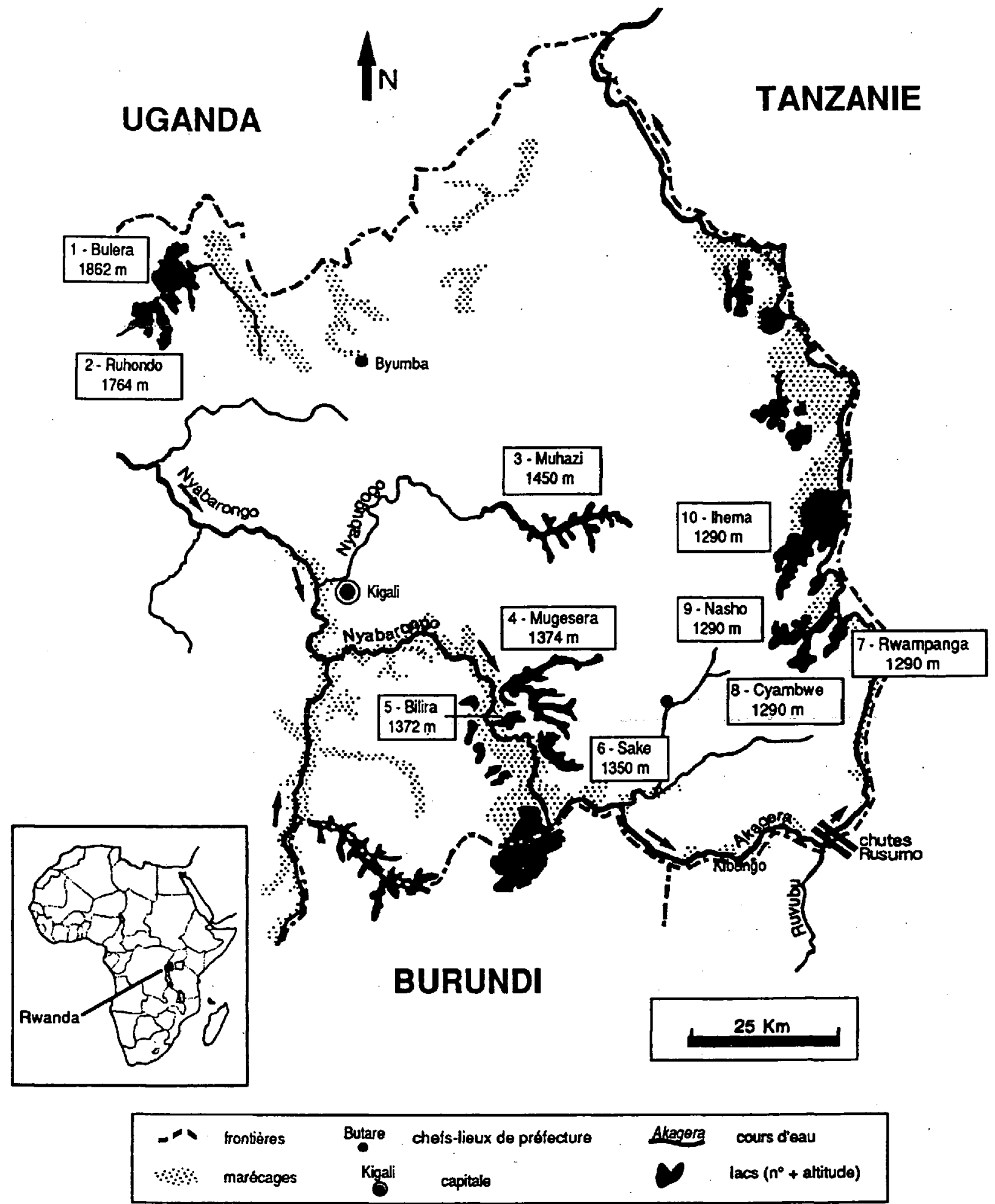

Fig. 1. Carte du Rwanda montrant la localisation des différents petits lacs, dont le lac Muhazi (3). (D'après Micha et Frank, 1991). Fig. 1. Map of Rwanda, showing the situation of various small lakes; among them, Lake Muhazi (3). 
partie ouest du lac, moins profonde que la zone est. En conséquence, le profil d'oxygène est le plus souvent contrasté, avec des sursaturations en surface et des concentrations chutant rapidement sous la zone de mélange. Les profils de $\mathrm{pH}$ sont similaires, avec des valeurs supérieures ả 8 en surface, descendant à des valeurs proches de 7 dans le fond du lac. Ces profils, typiques d'un lac eutrophe, ont été illustrés et discutés par Mukankomeje et al. (1993). Les eaux du Lac Muhazi sont bien minéralisées, la concentration totale en ions majeurs est de 9,6 méq. $1^{-1}$ avec une conductivité de l'ordre de $500 \mu \mathrm{S} . \mathrm{cm}^{-1}$ à $25^{\circ} \mathrm{C}$. L'ammonium est la principale source d'azote inorganique, avec des concentrations très faibles dans la zone photique, atteignant rarement $30 \mu \mathrm{g} . \mathrm{l}^{-1}$ à la surface après mélange. Le phosphore particulaire représente plus de $90 \%$ du phosphore total, qui atteint $46 \mu \mathrm{g} . \mathrm{l}^{-1}$, concentration typique d'un milieu eutrophe.

Une centaine de taxons phytoplanctoniques ont été identifiés au Lac Muhazi. Les Cyanobactéries sont représentées par 33 espèces regroupées au sein de 13 genres. C'est le groupe le plus abondant dans tous les échantillons (à toutes les saisons). Les formes coccoïdes dominent : Merismopedia spp., Microcystis spp., Aphanothece spp. ainsi que certaines formes filamenteuses : Anabaenopsis spp., Lyngbya spp. et Oscillatoria spp.. Pour les Dinophycées et les Cryptophycées, 5 genres ont été identifiés, cependant Ceratium et Peridinium sont les plus fréquemment observés, les autres genres se rencontrant de façon occasionnelle. Les Euglénophycées constituent le groupe le plus pauvre en espèces au Lac Muhazi : seuls Euglena, Phacus et Trachelomonas ont été identifiés et sont rares dans les échantillons. Parmi les 15 espèces de Diatomées déterminées, les plus fréquentes appartiennent aux genres Synedra, Nitzschia, Aulacoseira (anciennement Melosira), Achnantes et Cyclotella. Le groupe de Chlorophytes comprend le plus grand nombre de genres et d'espèces : 17 genres et 44 espèces de Chlorococcales et 3 genres appartenant aux Desmidiales (Cosmarium, Staurastrum et Staurodesmus). Les genres les plus fréquemment rencontrés sont des Chlorococcales : Monoraphidium, Tetraedron, Coelastrum, Scenedesmus... Cependant, malgré la relativement grande diversité, ce groupe n'est pas le plus abondant. En prenant comme référence la densité relative des " unités algales », les Cyanobactéries représentent 47-58 \%, les Chlorococcales 27-32 \%, les Diatomées
$15 \%$, les Euglénophycées et les Dinophycées font ensemble 1-3\%. L'estimation des biomasses à partir des biovolumes montre une prédominance très marquée de deux espèces : Microcystis aeruginosa (Kütz.) Kütz. représente plus de $75 \%$ et Ceratium hirundinella (O.F. Müll.) Duj. $9 \%$. La biomasse phytoplanctonique moyenne (50 $\mathrm{mg} \mathrm{Chl} a \cdot \mathrm{m}^{-2}$ ) sur la zone photique varie très peu en fonction des saisons ; elle est plus élevée à l'ouest, à cause d'une plus grande disponibilité des nutriments (voir Mukankomeje et al. 1993).

La faune piscicole du Lac Muhazi comprend très peu d'espèces indigènes : Haplochromis sp., Clarias liocephalus (Boulenger 1898), Barbus neumayeri (Fischer 1884) appelé aussi Barbus mohazicus (Pappenheym \& Boulenger 1914) et Barbus apleurogramma (Boulenger 1911). Les autres espèces ont été introduites : Tilapia rendalli (Boulenger 1898), Oreochromis spilurus niger (Günther 1894) et Oreochromis niloticus (Linné 1758) auraient été introduits en même temps vers les années 1930. Les deux dernières espèces semblent s'être hybridées, à tel point que peu d'Oreochromis niloticus de souche pure sont actuellement pêchés au Lac Muhazi. Afromastacembelus frenatus (Boulenger 1901) a été introduit vers 1960 ; les trois espèces de carpes, Cyprinus carpio (Linné 1758), Ctenopharyngodon idella (Valencienne 1844) et Hypophtalmichtys molitrix (Valencienne 1844) auraient été introduites au Lac Muhazi vers 1979. Clarias gariepinus (Burchell 1822), un silure de grande taille, a été introduit entre 1982 et 1984. Enfin, l'introduction de Protopterus aethiopicus (Heckel 1851) date de 1990. On peut ajouter à cette liste deux autres espèces dont on suspecte la présence au Lac Muhazi (Plisnier 1989) : Pseudocrenilabrus multicolor (Schoeller 1903) et Haplochromis burtoni (Günther 1893).

Des pêches expérimentales réalisées entre 1986 et 1987 ont permis d'estimer la proportion des espèces capturées (Plisnier 1989). Les Haplochromis viennent en tête avec $78 \%$ des captures pondérales, suivies par O. niloticus $(15,4 \%), T$. rendalli $(3,6 \%)$ et $\mathrm{Cla}$ rias gariepinus $(2,3 \%)$. Les statistiques de pêche récoltées auprès d'un échantillon de pêcheurs ont montré que les Tilapia spp. représentent $94 \%$ et $\mathrm{Cla}$ rias gariepinus $6 \%$ de leurs captures pondérales. L'exploitation des Haplochromis spp. pourrait contribuer à l'augmentation de la production piscicole du lac, car leur rendement maximum soutenu a été estimé à 24,4 kg.ha-1. an-1 (Plisnier 1990). 


\section{Matériel et Méthodes}

Les poissons ont été pêchés avec des filets maillants dont le vide de maille varie entre $20 \mathrm{~mm}$ et $60 \mathrm{~mm}$. Les filets, posés le soir, ont été relevés le matin. Pour les cycles de $24 \mathrm{~h}$, les filets ont été relevés toutes les 3 heures. Une autre partie des poissons a été pêchée à la senne tournante (lors des pêches des Haplochromis, pendant la nuit) pour avoir un nombre suffisant d'estomacs à analyser dans un intervalle de temps rapproché. Un échantillon de phytoplancton était prélevé en même temps pour comparer son contenu avec celui des contenus stomacaux.

Quatre campagnes de pêche ont été réalisées à la station de Nyarubuye, dans la partie ouest du lac (Fig. 2), à chaque saison. Trente estomacs (en moyenne) ont été examinés pour chaque campagne. Une autre campagne a été réalisée aux deux stations
(Nyarubuye, à l'ouest et Karambi, à l'est) pendant la grande saison des pluies, afin de comparer le régime alimentaire d' $O$. niloticus en fonction des stations. Enfin, un lot de 30 poissons, de taille comprise entre $90 \mathrm{~mm}$ et $140 \mathrm{~mm}$ (jeunes) et un autre de 30 , de taille supérieure à $140 \mathrm{~mm}$ (adultes), ont été retenus et leurs contenus stomacaux analysés pour vérifier l'alimentation en fonction de la taille des individus.

Chaque poisson a été pesé, mesuré (longueur totale et longueur standard), sexé et disséqué pour prélever l'estomac. On a déterminé la masse de ce dernier et un indice de réplétion (IR) lui a été attribué (IR $=0$ pour les estomacs vides et IR $=4$ pour les estomacs pleins). Les enveloppes stomacales ont été enlevées et pesées à leur tour afin de déterminer par différence la masse des contenus stomacaux. Ces contenus ont été conservés dans du formol à $10 \%$.

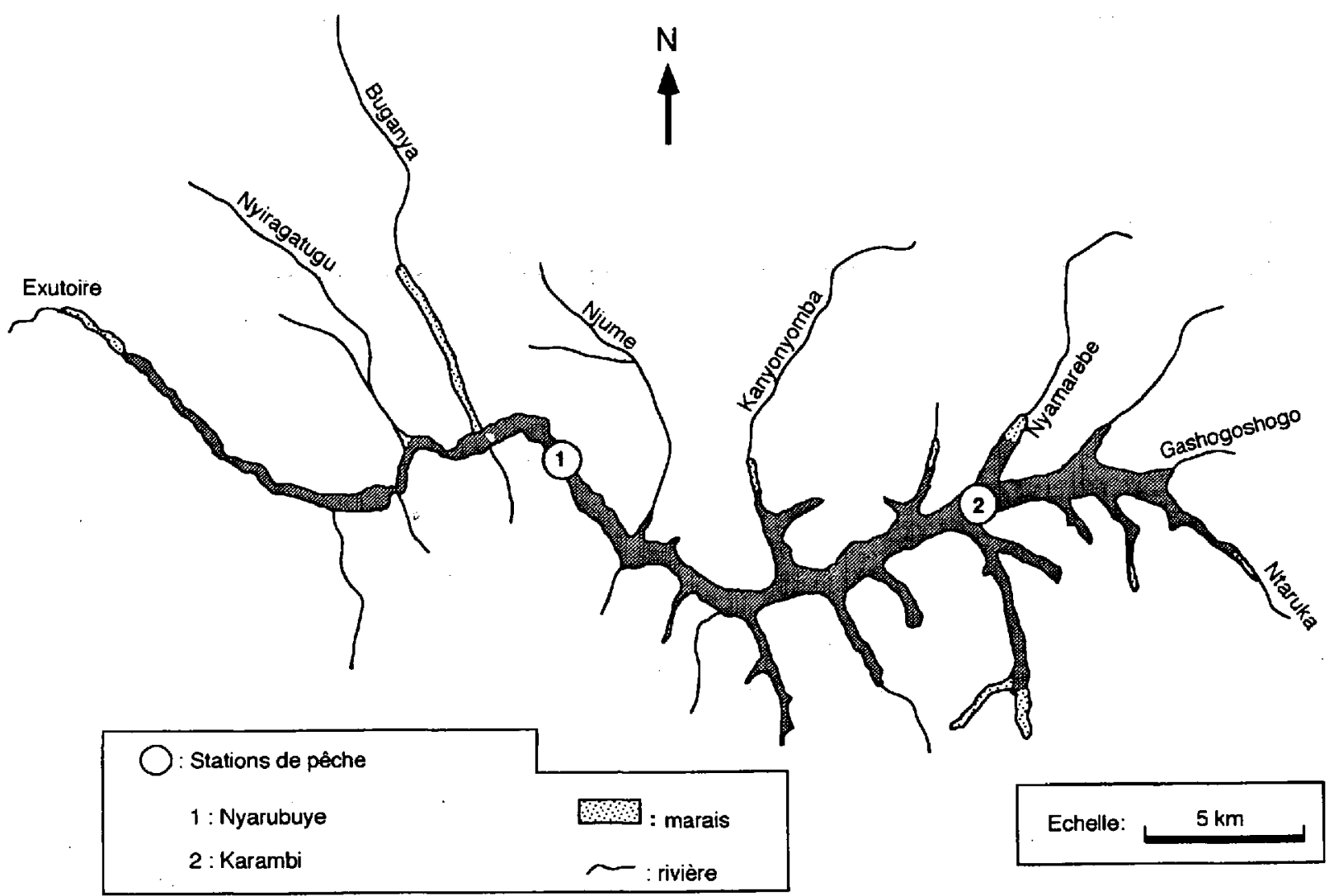

Fig. 2. Carte du lac Muhazi montrant la localisation des stations de pêches. (D'après Plisnier 1989).

Fig. 2. Map of lake Muhazi, with the sites of the sampling stations. 
L'examen des contenus stomacaux a été réalisé après dilution adéquate et ajout de $1 \mathrm{ml}$ de Lugol. Les algues ont été identifiées en microscopie optique, à l'aide des ouvrages de Bourrelly $(1966,1968,1970)$, Compère $(1974,1975,1976,1977)$, Iltis (1980) et Komarek \& Fott (1983). Le volume de la plupart des taxons a été calculé par assimilation à une forme géométrique simple (sphère, cube, cylindre...), selon Vollenweider (1974) et Sournia (1978), à partir d'un échantillon de 25 individus par taxon. Une cellule de Bürker a été utilisée pour le comptage du plancton des contenus stomacaux. Vu l'impossibilité d'analyser tout le contenu stomacal (et au total 118 contenus stomacaux), nous avons utilisé une procédure empirique pour sous-échantillonner. Cette procédure est préconisée dans le cas où la distribution des algues dans l'estomac est inconnue ou hétérogène (Abel 1973). C'est ainsi que pour chaque contenu stomacal, nous avons trouvé que trois sous-échantillons nous permettaient de rencontrer le maximum de taxons présents dans l'estomac. Pour chaque sous-échantillon, un comptage de 18 carrés de la cellule de Bürker s'est révélé largement suffisant. La densité des unités algales dans les suspensions de contenus stomacaux a été calculée par comptage de trois échantillons totalisant $0.2 \mathrm{~mm}^{3}$ environ.

Une analyse en composantes principales (ACP) a été appliquée sur les matrices suivantes : une de dimension « $73 * 118$ » (73 espèces phytoplanctoniques et 118 contenus stomacaux) pour l'analyse du régime alimentaire en fonction des saisons et de deux autres de même dimension, « $73^{*} 60$ » (73 espèces phytoplanctoniques et 60 contenus stomacaux) pour l'examen du régime alimentaire en fonction des stations et en fonction de la taille des poissons. Dans les trois cas, les variables ont subi une transformation logarithmique, puis ont été réduites (soustraction de la moyenne et division par l'écart-type) afin de les rendre équivalentes au départ.

Pour tous les contenus stomacaux analysés, nous n'avons retenu que les taxons algaux dont l'abondance est supérieure à 50 individus. $\mathrm{ml}^{-1}$. Le programme d'analyse multivariée utilisé est inspiré de Lebart et al. (1979).

\section{Expression des résultats}

Quatre indices ont été retenus pour décrire le régime alimentaire :
- l'indice d'occurrence (Io), qui tient compte du nombre d'estomacs $\left(\mathrm{N}_{\mathrm{i}}\right)$ dans lesquels une proie $\mathrm{i}$ est observée ;

- l'indice d'abondance (Iab) qui considère uniquement le nombre d'observations d'une catégorie d'aliments par rapport au nombre total d'individus pour l'ensemble de l'échantillon ;

- l'indice volumétrique (Iv) qui tient compte du biovolume des proies et permet d'évaluer la biomasse relative des différentes proies ;

- l'indice alimentaire ou de dominance (IA) de Lauzanne (1976) ; ce dernier tient compte à la fois de l'indice d'occurrence de chaque proie (Io) et de son indice volumétrique (Iv). Lauzanne (1976) a proposé une classification des valeurs d'indices : lorsque IA est supérieur à 50 , la proie est dite dominante ; s'il est compris entre 50 et 25 , elle est dite essentielle. La proie est considérée comme importante lorsque IA est entre 25 et 10 . Enfin, la proie est dite secondaire si IA est inférieur à 10. Par rapport aux autres indices, celui-ci est utile quand on veut comparer les régimes alimentaires d'une même espèce en fonction de la taille, des saisons et du biotope.

Ces différents indices sont calculés de la manière suivante :

$$
\begin{aligned}
& \text { soit } \quad \mathrm{N}_{\mathrm{i}}=\text { nombre d'estomacs où la proie } \mathrm{i} \\
& \mathrm{N}_{\mathrm{t}}=\text { nombre total d'estomacs examinés } \\
& \mathrm{n}_{\mathrm{i}}=\text { nombre d'individus de la proie } \mathrm{i} \\
& \stackrel{p}{\sum} n_{i}=\text { nombre total d'individus des } p \\
& \mathrm{i}=1 \\
& v_{\mathbf{i}}=\text { biovolume de la proie } \mathrm{i} \\
& \sum_{\mathrm{i}=1}^{\mathrm{p}} \mathrm{n}_{\mathrm{i}} \mathrm{v}_{\mathrm{i}}=\text { biovolume total des p proies }
\end{aligned}
$$

$$
\begin{aligned}
\text { Alors } \quad \text { Io } & =100\left(\mathrm{~N}_{\mathrm{i}} / \mathrm{N}_{\mathrm{t}}\right) \\
\text { Iab } & =100\left(\mathrm{n}_{\mathrm{i}} / \sum_{\mathrm{i}=1}^{\left.\sum_{\mathrm{n}_{\mathrm{i}}}\right)}\right. \\
\mathrm{Iv} & =\left(\mathrm{n}_{\mathrm{i}} \mathrm{v}_{\mathrm{i}} / \sum_{\mathrm{i}=1}^{\left.\sum_{\mathrm{n}_{\mathrm{i}} \mathrm{v}_{\mathrm{i}}}\right) 100}\right. \\
\text { IA } & =(\text { IoIv }) / 100
\end{aligned}
$$

L'indice d'électivité $\left(E_{i}\right)$ d'Ivlev (1961) a été utilisé pour apprécier une sélection éventuelle du 
phytoplancton par $O$. niloticus. Cet indice se définit comme :

$$
E_{i}=\left(r_{i}-p_{i}\right) /\left(r_{i}+p_{i}\right)
$$

$r_{i}=$ abondance relative $d u$ taxon $i$ dans les contenus stomacaux (indice d'abondance du taxon i)

$\mathrm{p}_{\mathrm{i}}=$ abondance relative $\mathrm{du}$ taxon $\mathrm{i}$ dans le phytoplancton du lac

La sélection est dite totale (positive) si $\mathrm{E}_{\mathrm{i}}$ est égale à 1 ; elle est dite négative (pas de consommation du taxon i) si $E_{i}$ vaut - 1 . Le poisson a un comportement indifférent vis-à-vis du taxon i si $E_{i}$ vaut 0 .

La ration journalière a été estimée à l'aide de :

- l'équation de régression linéaire simple utilisée par Moriarty \& Moriarty (1973) pour la même espèce, équation uniquement applicable aux poissons dont la taille est supérieure à $50 \mathrm{~mm}$. Elle tient compte de la masse du poisson :

$$
\mathrm{Y}=271+13,3 \mathrm{~W}
$$

avec $\mathrm{Y}=$ masse $\mathrm{du} \cdot$ phytoplancton ingéré (matière sèche) par poisson par jour (mg.j-1) ;

$\mathrm{W}=$ masse $\mathrm{du}$ poisson frais $(\mathrm{g})$;

- l'équation de régression linéraire multiple, utilisée par Palomarès \& Pauly (1989), qui tient compte a la fois de la masse du poisson, des conditions de l'environnement et des caractéristiques propres de l'espèce, dont son indice d'activité, le type de nourriture consommée ainsi que de son statut taxonomique. Après simplification, l'équation établie pour O. niloticus (Palomarès \& Pauly 1991) est de la forme suivante :

$$
\begin{aligned}
\log \mathrm{Rd} & =-1,406+0,782 \log \mathrm{W}+0,571 \log \mathrm{T} \\
\text { où } \mathrm{Rd} & =\text { ration journalière }(\mathrm{g}) \\
\mathrm{W} & =\text { masse du poisson frais }(\mathrm{g}) \\
\mathrm{T} & =\text { la température de l'eau }\left({ }^{\circ} \mathrm{C}\right)
\end{aligned}
$$

- le logiciel Maxims, mis au point par Jarre et al. (1991), qui se base sur l'évolution des contenus stomacaux échantillonnés sur des cycles de $24 \mathrm{~h}$. Ce logiciel offre l'avantage d'estimer le moment de l'alimentation ainsi que la quantité de nourriture consommée par unité de biomasse $(Q / B)$.

Le calcul de la quantité de phytoplancton consommée par unité de biomasse $(Q / B)$ est basée sur l'équation de Pauly (1986) qui nécessite les paramètres de la croissance et de la mortalité en milieu naturel ainsi que les coefficients de conversion de la nourriture et(ou) les rations journalières pour une masse donnée, obtenus à partir d'expériences de laboratoire et/ou les observations de terrain. Pour $O$. niloticus du Lac Muhazi, nous avons considéré :

- les valeurs suivantes de l'équation de Von Bertalanffy, le poids maximum $\left(\mathrm{V}_{\infty}\right)$ de $1300 \mathrm{~g}$, le coefficient de croissance $(\mathrm{K})$ de 0,33 .an-1, l'âge à la taille $O$ (to) de 0,12 an et le coefficient $b$ de 3,21 ;

- le taux de mortalité (M) de 0,77.an-1 calculé à partir de l'équation de Pauly (1980) :

$\log M=0,0066-0,279 \log L \infty+0,065431$ $\log K+0,4631 \log T$, en considérant une longueur standard à l'infini (Loc) de $38,5 \mathrm{~cm}$, une tempéra-

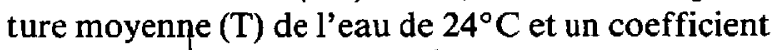
de croissance $K$ de $0,33 . \mathrm{an}^{-1}$;

$-B$, variable qui relie le coefficient de conversion de la nourriture $\left(\mathrm{k}_{1}\right)$ à la masse du poisson (W) de la façon suivante $: k_{1}=1-(W / W \infty) \beta$, a été estimé d'après Jarre et al. (1991), à l'aide du programme Maxims, à partir de là ration journalière et de la masse individuelle de chaque poisson. La valeur obtenue est de 0,0155 .

\section{Résultats}

La figure 3 présente les résultats principaux de l'examen des contenus stomacaux pour les grandes catégories taxonomiques, aux différentes saisons. Il en ressort clairement que le phytoplancton présente la fraction la plus abondante, le zooplancton et les macrophytes étant peu représentés (Fig. 3 A). La figure 3 B permet de situer les préférences alimentaires d' $O$. niloticus parmi les différents groupes algaux. L'indice d'occurrence varie entre $100 \%$ pour certaines Cyanobactéries telles Merismopedia tenuissima Lemm. Microcystis holsatica Lemm. et $1,3 \%$ pour une Cryptophycée, Cryptomonas sp. Les différents indices montrent que les Cyanobactéries constituent une proie importante pour $O$. niloticus au Lac Muhazi (Io $=69,9 \%$, Iab $=64,3 \%$; Iv $=83,1 \%$ et IA $=58,1)$. Les Dinophycées viennent en deuxième position au point de vue biomasse $(\mathrm{Iv}=14,2 \%)$ à cause de $C$. hirundinella qui représente un indice volumétrique de 13,04 \%. Parmi les Cyanobactéries, Microcystis aeruginosa (Kütz.) Kütz. a un indice d'occurrence de $94,6 \%$, un indice volumétrique de $81,5 \%$ et un indice alimentaire de 77,1 , alors que son indice d'abondance est seulement de $3,9 \%$. Ainsi, cette espèce est la seule proie dominante $\left(\mathrm{IA}_{\mathrm{A}}>50\right)$ et toutes les autres sont des proies secondaires. 
A

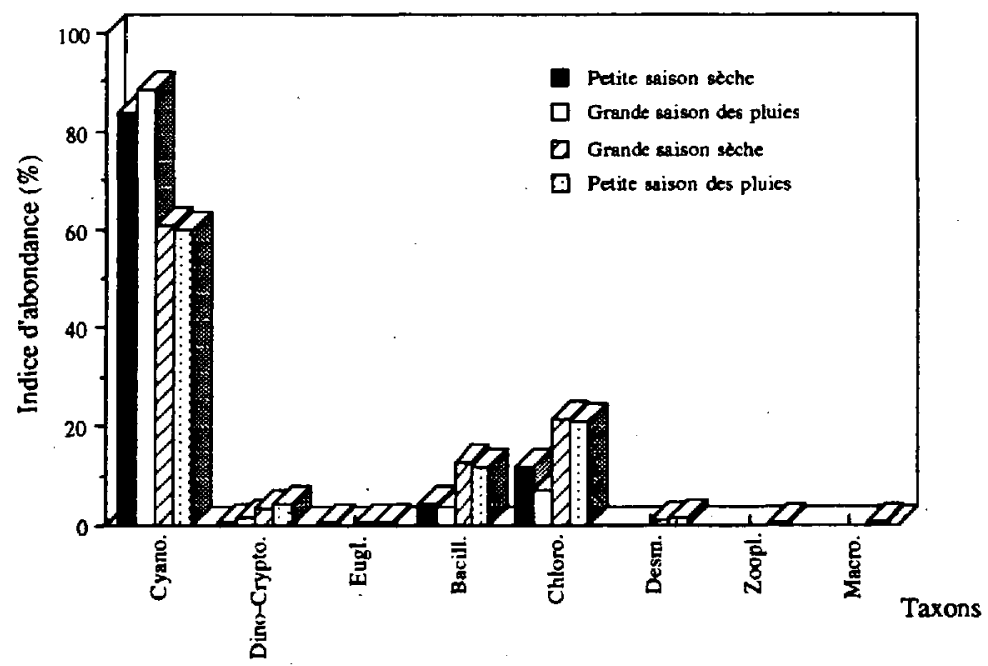

B

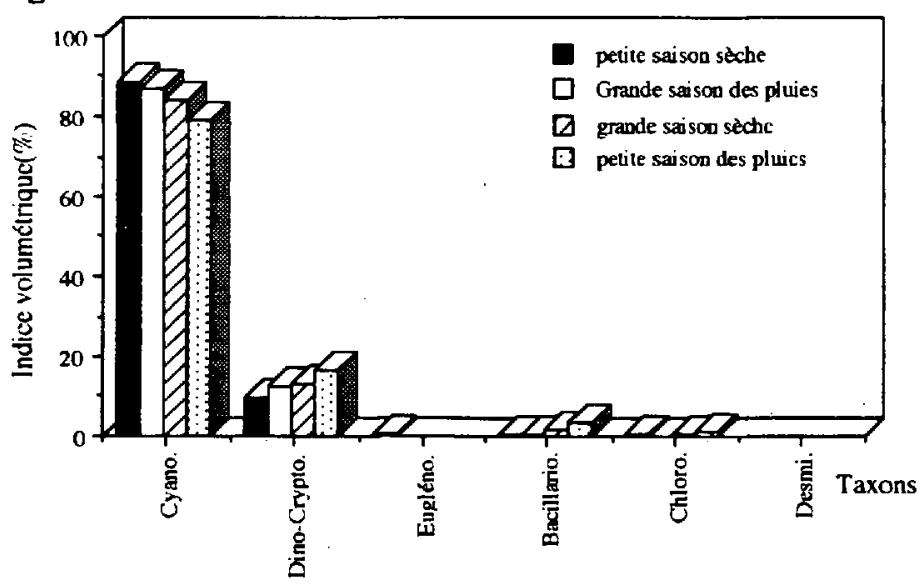

Fig. 3. Répartition des indices d'abondance (A) et volumétriques (B) des grandes catégories d'aliments dans les contenus stomacaux d'O. niloticus du Lac Muhazi, à la station ouest au cours des différentes saisons, de décembre 1989 à septembre 1990.

Fig. 3. Distribution of abundance (A) and volumetric (B) indices of the main prey categories in the stomach contents of $O$. niloticus in lake Muhazi, at the western station during different seasons, from December 1989 to September 1990.

L'alimentation d'O. niloticus varie sensiblement en fonction des saisons. Ceci est confirmé par l'ACP qui montre une assez bonne discrimination des échantillons suivant la saison (Fig. 4). Cependant la figure 3 montre que, en toutes saisons, les
Cyanobactéries sont les mieux représentées : l'indice d'abondance (Iab) varie entre $59,8 \%$ et $87,9 \%$ (Fig. 3A) et l'indice volumétrique (Iv) entre 78,7\% et 88,3\% (Fig. 3B). Quelques espèces dont l'indice d'abondance esí supérieur à $1 \%$ pour au moins une 
F2

Fig. 4. Régime alimentaire d'O. niloticus du lac Muhazi : comparaison entre les saisons par l'analyse en composantes principales pour les poissons pêchés à la station ouest de décembre 1989 à septembre 1990 (PSS = petite saison sèche ; GSP = grande saison des pluies ; GSS = grande saison sèche ; PSP = petite saison des pluies).

Fig. 4. Diet of $O$. niloticus in lake Muhazi : comparaison between seasons by principal components analysis for fishes caught at the western station from December 1989 to September 1990 (PSS = small dry season ; GSP = great rainy season ; GSS = great dry season and PSP $=$ small rainy season).

des saisons, ont été retenues pour déceler l'origine de la variation saisonnière mise en évidence par l'ACP alors que la composition du phytoplancton du lac ne montre pas de variation temporelle significative (Mukankomeje et al. 1993). Il s'agit essentiellement des Cyanobactéries : Aphanothece spp. (Apha), Lyngbya contorta Lemm. (Lcon), L. limnetica Lemm. (Llim), Lyngbya spp. (Lspp), Merismopedia punctata Meyen (Mepu), Merismopedia tenuissima (Mete), Microcystis aeruginosa (Miae), Microcystis densa G.S. West (Mide), Microcystis holsatica Lemm. (Miho), Microcystis incerta (Lemm.) Lemm. (Miin), Oscillatoria spp. (Ospp), Synechocystis leopoliensis (Racib.) Kom. (Sleo), et les Nostocales indéterminés (Nost). Les autres groupes sont représentés par très peu d'espèces : Ceratium hirundinella (Cehi), une Dinophycée, deux Bacillariophycées: Cyclotella (Cycl), Nitzschia (Nspp) et trois .Chlorophycées : Dictyosphaerium spp. (Dict), Didymocystis bicellularis (Chod) Kom.
(Dbic) et Monoraphidium tortile (W.\& G.S. West) Kom.-Legn (Moto). La distribution des indices d'abondance de ces espèces pour les poissons pêchés pendant la petite saison sèche et la grande saison des pluies est différente de celle obtenue à partir des poissons prélevés pendant la grande saison sèche et la petite saison des pluies. En effet, pour les poissons de la petite saison sèche et de la grande saison des pluies, quelques espèces sont très abondantes dans les contenus stomacaux : Merismopedia tenuissima (Mete), Microcystis holsatica (Miho), Oscillatoria spp. (Ospp) ont un indice d'abondance supérieur à $10 \%$ (Fig. SA). Par contre, pour les contenus stomacaux des poissons pêchés pendant la grande saison sèche et la petite saison des pluies, toutes les espèces prennent une part plus ou moins égale dans l'alimentation. Merismopedia tenuissima (Mete) reste la plus importante avec un indice d'abondance de $8 \%$ en petite saison des pluies (Fig. 5B). 
A

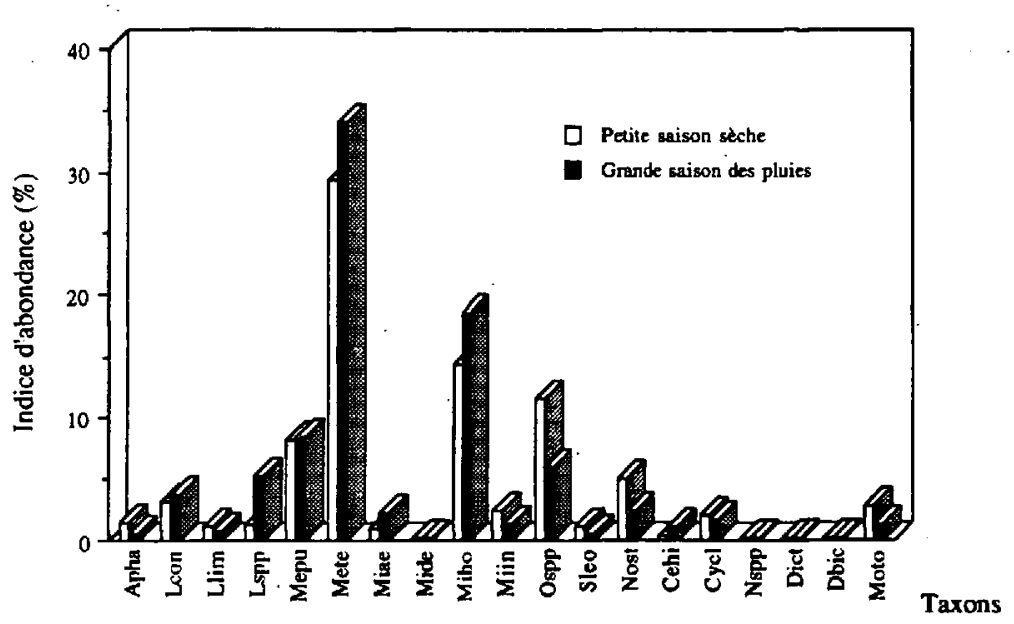

$\mathrm{B}$

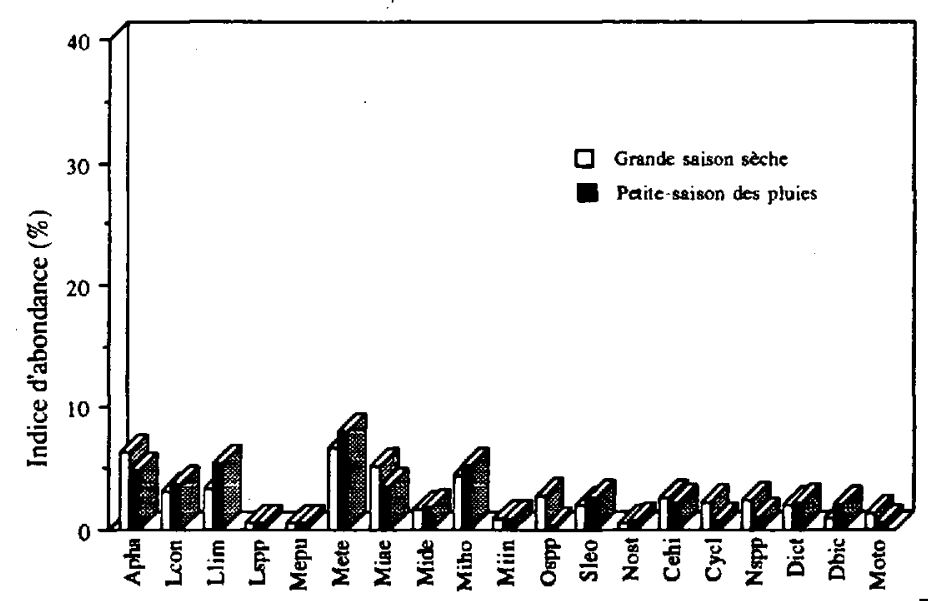

Taxons

Fig. 5. Comparaison des indices d'abondance des principales espèces phytoplanctoniques dans les contenus stomacaux d'O. niloticus du Lac Muhazi, à la station ouest, pendant la petite saison sèche et la grande saison des pluies (A) et pendant la grande saison sèche et la petite saison des pluies (B) 1990. Voir dans le texte la signification des abréviations.

Fig. 5. Comparison of abundance indices of the main phytoplankton species in the stomach contents of $O$. niloticus in lake Muhazi, at the western station during the small dry season and the great rainy season (A) and during the great dry season and the small rainy season (B) 1990. See text for the meaning of the abbreviations.

Ces différences en fonction des saisons sont confirmées par les coefficients d'électivité d'Ivlev calculés pour quelques espèces. Nous avons constaté que, pendant la petite saison sèche et la grande saison des pluies (Fig. 6A), seules quelques espèces, surtout des Cyanobactéries (Lyngbya contorta, Merismopedia spp., Microcystis spp., Oscillatoria spp.) et $C$. hirundinella sont sélectionnées positivement par le poisson. Par contre, pendant la grande saison sèche et la petite saison des pluies (Fig. 6B), 


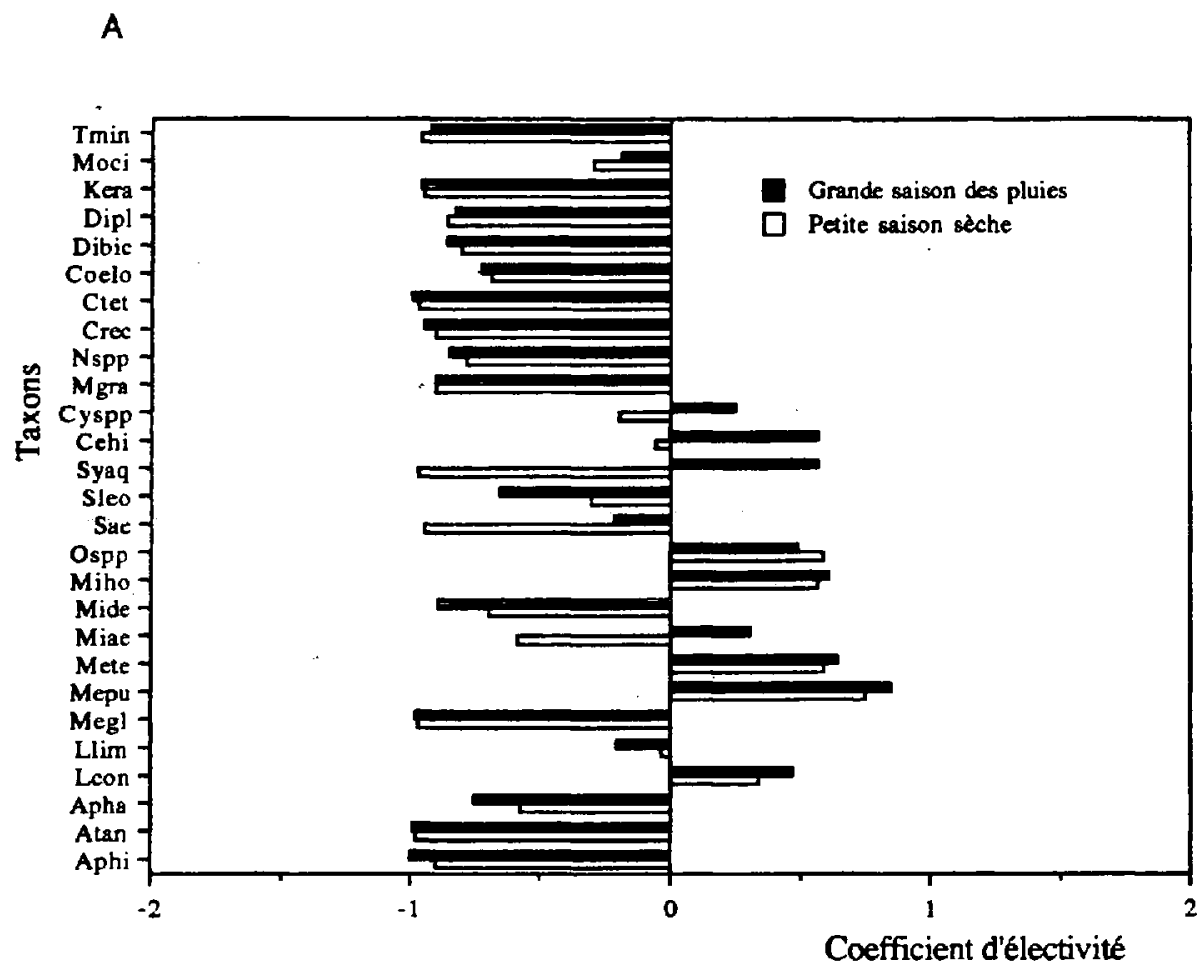

B

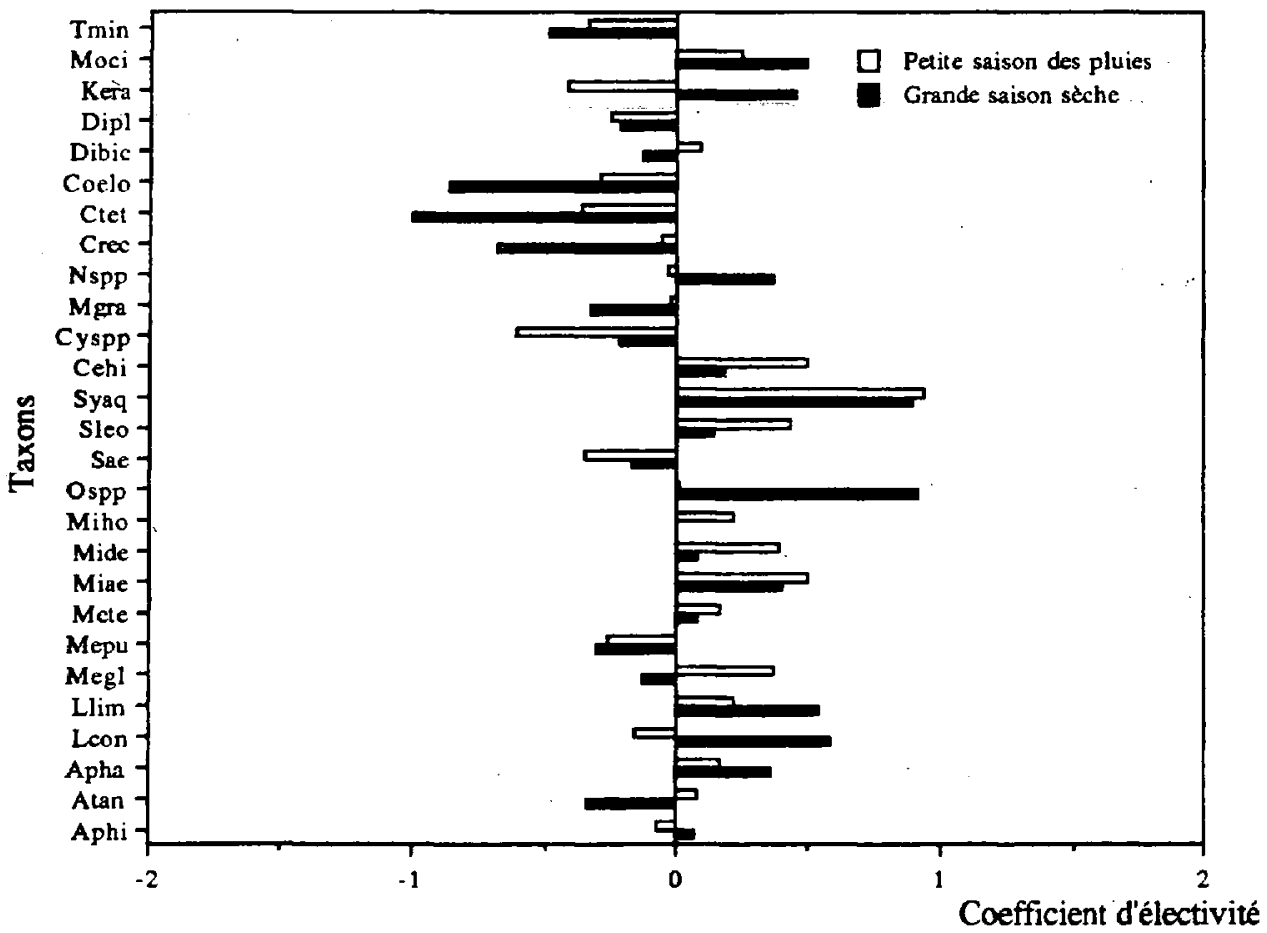

Fig. 6. Coefficient d'électivité d'Ivlev de certains taxons abondants dans le phytoplancton du lac et des contenus stomacaux d'O. niloticus du lac Muhazi, à la station ouest, pendant la petite saison sèche et la grande saison des pluies (A) et pendant la grande saison sèche et la petite saison des pluies (B) 1990.

Fig. 6. Ivlev's electivity index of some phytoplankton taxa abundant in the lake water and in the stomach contents of $O$. niloticus, at the western station during the small dry season and the great rainy season (A) and during the great dry season and the small rainy season (B) 1990. 
la majorité des espèces, y compris des Chlorococcales (Monoraphidium circinale (Nyg.) Nyg., Keratococcus) et les diatomées (Nitzschia spp.) sont sélectionnées par le poisson.

L'analyse en composantes principales montre que le régime alimentaire de $O$. niloticus est différent en fonction des stations (Fig. 7). Une interprétation détaillée a permis de mettre en évidence que la différence réside dans le nombre des colonies et le nombre des filaments de quelques Cyanobactéries trouvées dans les contenus stomacaux. C'est ainsi que Lyngbya spp. et Oscillatoria spp. sont abondants dans les contenus stomacaux des poissons pêchés à la station est, alors que les Microcystis spp. sont plus abondants dans les contenus stomacaux de la station ouest.

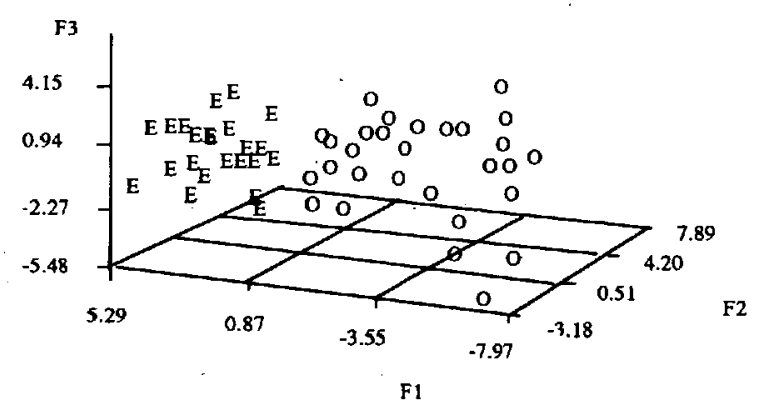

Fig. 7. Régime alimentaire d'O. niloticus du lac Muhazi : comparaison entre les stations est (E) et ouest (O) par l'analyse en composantes principales sur les contenus stomacaux des poissons pêchés en mars-avril 1990.

Fig. 7. Diet of $O$. niloticus in lake Muhazi : comparison between the eastern $(E)$ and the western $(O)$ station by the principal components analysis on fishes caught in March-April 1990.

Pour les poissons de taille comprise entre $90 \mathrm{~mm}$ et $140 \mathrm{~mm}$ (jeunes) et pour ceux supérieurs à $140 \mathrm{~mm}$ (adultes), le phytoplancton était abondant dans tous les contenus stomacaux. Les Chlorophytes sont plus consommés par les adultes (Iab = $11,5 \%)$ que par les jeunes ( $\mathrm{Iab}=6,7 \%$ ) et les Cyanobactéries sont aussi bien consommées par les adultes $(\mathrm{Iab}=83,5 \%)$ que par les jeunes (Iab = $88,9 \%$ ). La différence entre les deux groupes (jeunes et adultes) est mise en évidence par l'ACP (Fig. 8). La ségrégation assez marquée entre jeunes et adultes provient de la quantité de colonies et de filaments dénombrés : ainsi, $M$. aeruginosa est plus abondant dans les contenus stomacaux des poissons adultes que dans les contenus stomacaux des jeunes poissons.

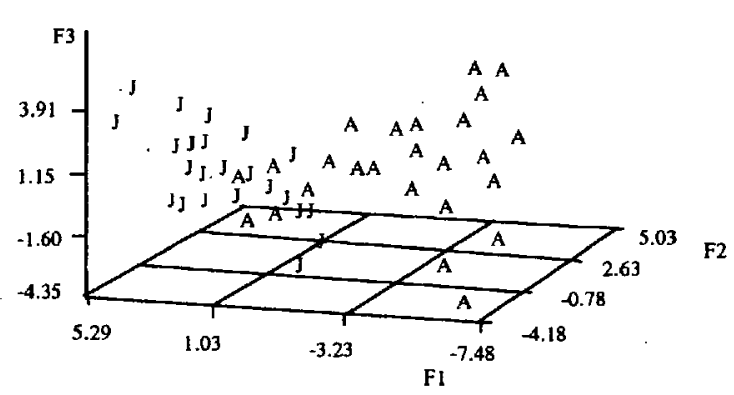

Fig. 8. Régime alimentaire d'O. niloticus du lac Muhazi : comparaison entre les poissons adultes (A) et les jeunes (J) pêchés à la station ouest en mars-avril 1990, par l'analyse en composantes principales.

Fig. 8. Diet of $O$. niloticus in lake Muhazi : comparison between the adults specimen (A) and the juveniles ( $\mathrm{J}$ ) by principal components analysis.

Enfin, le moment d'alimentation a été déterminé grâce au suivi du remplissage de l'estomac au cours de cinq cycles de $24 \mathrm{~h}$, réalisés par tranches de $3 \mathrm{~h}$ (20 poissons par tranche) pendant la grande saison des pluies de l'année 1990 aux deux stations. Les résultats montrent que $O$. niloticus se nourrit entre $6 \mathrm{~h}$ et $21 \mathrm{~h}$ (Fig. 9). Suivant la méthode de calcul, la ration journalière de phytoplancton frais est de $21,2 \mathrm{~g} . \mathrm{j}^{-1}$ (régression linéaire multiple), de $22,2 \mathrm{~g} \cdot \mathrm{j}^{-1}$ (régression linéaire simple) et de $26,5 \mathrm{~g} . \mathrm{j}^{-1}$ (logiciel Maxims). En se basant sur cette dernière valeur, la quantité consommée par unité de biomasse $(Q / B)$ est de $25,7$. an-1. $^{-1}$.

\section{Discussion et conclusion}

Le phytoplancton est très abondant dans les contenus stomacaux d'O. niloticus. Les Cyanobactéries sont prédominantes en nombre et en biomasse tout comme dans l'eau du lac. $M$. aeruginosa représente plus de $80 \%$ de l'indice volumétrique quelles que soient la saison, la station et la taille des poissons ( $\geqslant 90 \mathrm{~mm}$ ). Les valeurs des différents indices calculés sont comparables à celles obtenues pour la même espèce, pour les individus de même taille et obtenus par les mêmes techniques de pêche dans d'autres lacs africains. C'est ainsi qu'au Lac George (Uganda), les Cyanobactéries constituent plus de $70 \%$ du régime alimentaire d'O. niloticus (Ganf 


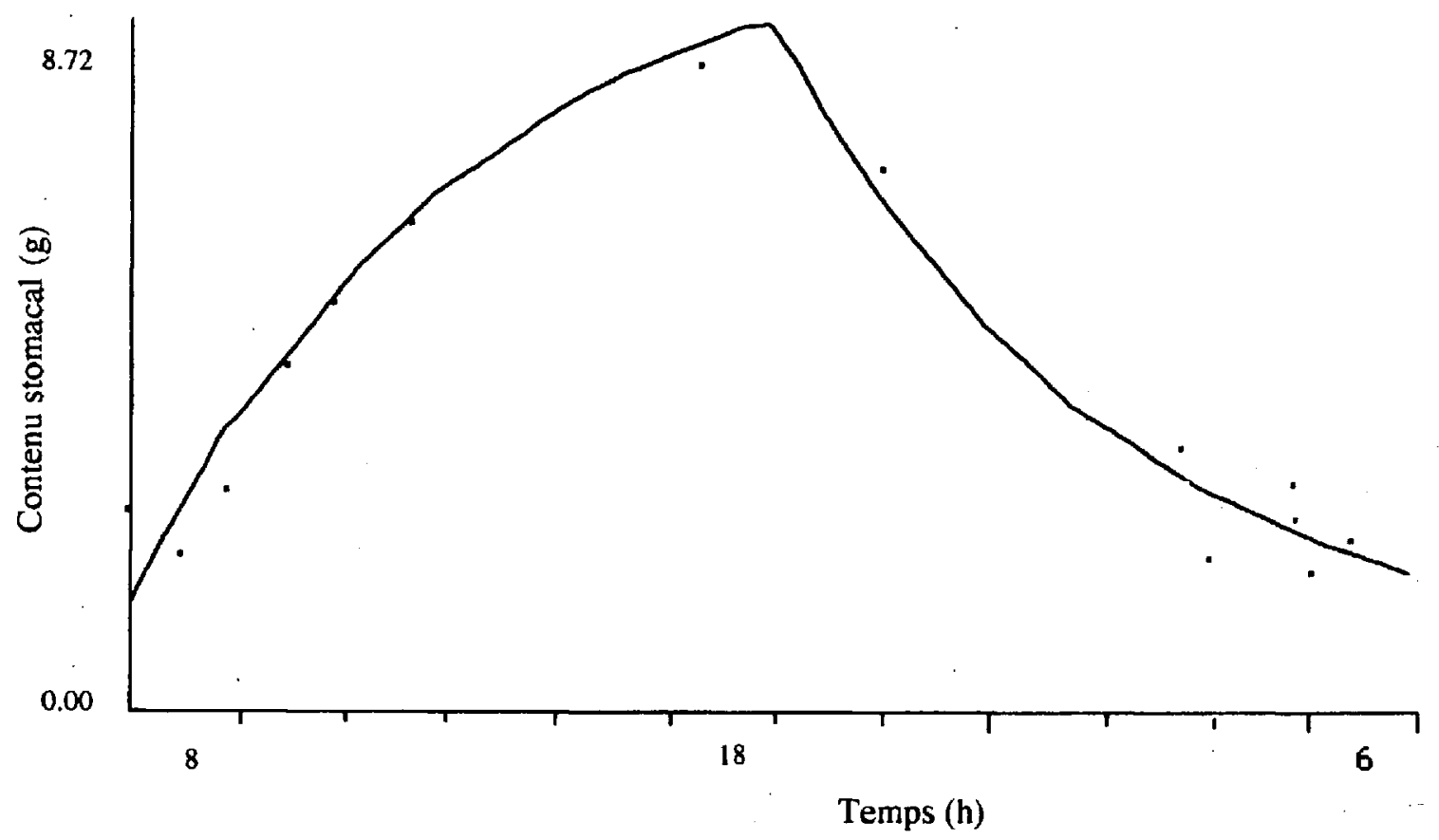

Fig. 9. Régime alimentaire d' $O$. niloticus du lac Muhazi : estimation de la ration journalière à partir des contenus stomacaux des poissons pêchés au cours des cycles de 24 h (février-avril 1990).

Fig. 9. Diet of $O$. niloticus in lake Muhazi : estimation of daily ration from the stomach contents of fishes caught during $24 \mathrm{~h}$ cycles (February-April 1990).

1972). Au Lac Awasa (Ethiopie), par contre, les Cyanobactéries représentent $28,1 \%$ et Botryococcus (taxon très abondant dans le lac) $67,2 \% \mathrm{du}$ régime alimentaire (Getachew \& Fernando 1989). Ces observations confirment bien le régime phytoplanctonophage d'O. niloticus, qui possède des enzymes digestives qui lui permettent de bien digérer les algues (Al-Hussaini \& Kholy 1954, Moriarty 1973, Trewavas 1983). Ce même type de régime alimentaire se rencontre chez de nombreux tilapias, comme par exemple Sarotherodon melanotheron (Ugwumba \& Adebis 1992). Chez cette espèce, au Lac Awba (Nigeria), il existe une différence de régime alimentaire entre les juvéniles, plutôt omnivores, et les adultes, essentiellement phytoplanctonophages et détritivores. Des observations similaires sont faites par Ufodike \& Wada (1991) en étang d'élevage : les jeunes sujets, jusqu'à $25 \mathrm{~mm}$, préfèrent le zooplancton à une alimentation artificielle. Une différence aussi nette ne se retrouve pas chez les $O$. niloticus du Lac Muhazi, ce qui est peu surprenant si l'on sait qu'à la taille de $90 \mathrm{~mm}$, le développement branchial est complètement achevé chez cette espèce (Northcott \& Beveridge 1988), ce qui leur permet de consommer principalement du phytoplancton. De plus, Tudorancea et al. (1988) ont trouvé en abondance des Cyanobactéries et des Diatomées dans les contenus stomacaux des jeunes O. niloticus de $30 \mathrm{~mm}$. Il faut enfin souligner, comme le suggèrent Ugwumba \& Adebis (1992), que le régime alimentaire des adultes et des juvéniles peut être influencé par leur habitat : les juvéniles, vivant en zone littorale, ont à leur disposition une nourriture plus variée que les adultes vivant au large et exploitant surtout le phytoplancton pélagique.

Il existe, au Lac Muhazi, une faible variation saisonnière du régime alimentaire, qui ne correspond pas à une variation similaire de la composition du phytoplancton dans le milieu. De même, on a pu mettre en évidence une variation stationnelle significative, qui résulte du fait que les colonies et les 
filaments de certains taxons (Microcystis spp., Oscillatoria spp., Lyngbya spp. et $C$. hirundinella) sont mieux représentés dans la partie ouest du lac (Mukankomeje 1992).

O. niloticus semble consommer de façon préférentielle des taxons algaux de grande taille. Ainsi, les Cyanobactéries filamenteuses et coloniales ainsi que Ceratium sont sélectionnés passivement. Ceci concorde avec les observations de Northcott et al. (1991) : ils ont constaté (en laboratoire) qu'O. niloticus a un taux de filtration et d'ingestion élevé lorsqu'il est nourri d'algues coloniales. Suivant les observations de Moriarty (1973), travaillant sur la même espèce au Lac George, la taille des proies n'est pas seule en cause pour la sélection, un pouvoir d'adhérence différentiel des algues sur du mucus couvrant les branchiospines joue également un rôle.

Les différentes techniques employées (indice de réplétion, évolution des contenus stomacaux prélevés sur des cycles de $24 \mathrm{~h}$ ) montrent qu'O. niloticus se nourrit entre $6 \mathrm{~h}$ et $21 \mathrm{~h} 30$. Les estimations de la ration journalière varient entre $21 \mathrm{~g} . \mathrm{j}^{-1}$ et $26,5 \mathrm{~g} . \mathrm{j}^{-1}$ de phytoplancton frais. Cette dernière valeur est comprise entre celles obtenues au Lac George (9,5 g.j-1) et au Lac Awasa (39,3 g.j-1) pour la même espèce (Palomarès 1991).

La quantité de phytoplancton consommée par unité de biomasse (Q/B) est de 25,7.an.-1. La valeur estimée à l'aide de l'équation établie par Palomarès (1991) pour les tilapias $(\log Q / B=0,791-$ $0,165 \log \mathrm{W} \infty+0,759 \log \mathrm{T} ; \mathrm{T}=$ température de l'eau en ${ }^{\circ} \mathrm{C}$ ) est de $23,4 . \mathrm{an}^{-1}$ soit un $\mathrm{Q} / \mathrm{B}$ moyen de 24,5. an-1. Ces deux valeurs sont proches et restent inférieures à celle estimée au Lac Awasa $\left(49,9 . \mathrm{an}^{-1}\right)$ et supérieures à celles obtenues par les mêmes techniques au Lac George (12,8.an-1) et au Lac Turkana $\left(17,2 . \mathrm{an}^{-1}\right)$ (Palomarès 1991). La valeur relativement élevée de $\mathrm{Q} / \mathrm{B}$ obtenue au Lac Muhazi est sans doute due à la faible biomasse d' $O$. niloticus.

Enfin, nous avons tenté d'estimer la part de la production primaire consommée par $O$. niloticus. Pour ce faire, nous considérons une biomasse maximale de tilapias d'environ $30 \mathrm{~kg} \cdot \mathrm{ha}^{-1}$ (Plisnier, 1990) et un poids moyen individuel de $180 \mathrm{~g}$, soit une densité moyenne de poissons de $167 \mathrm{ha}^{-1}$. Sachant qu'un individu ingère $26 \mathrm{~g} . \mathrm{j}^{-1}$, soit $2,86 \mathrm{~g} \mathrm{C} . \mathrm{j}^{-1}$ (conversion d'après Lind, 1979) on peut estimer que la population d' $O$. niloticus consomme environ $478 \mathrm{~g} \mathrm{C} \mathrm{ha}^{-1} \cdot \mathrm{j}^{-1}$. Cette valeur représente quelque
$6 \%$ de la production primaire nette moyenne (7 $900 \mathrm{~g} \mathrm{C} \mathrm{ha}^{-1} . \mathrm{j}^{-1}$, d'après Mukankomeje et al. 1993). Par contre, en appliquant directement l'équation de Palomarès \& Pauly (1991) à la biomasse de tilapias, on obtient une consommation de phytoplancton frais de $0,78 \mathrm{~kg} \cdot \mathrm{ha}^{-1} . \mathrm{j}^{-1}$, soit moins de $1 \%$ de la production primaire nette moyenne.

L'objectif initial du présent travail est d'identifier les causes de la faible production piscicole du Lac Muhazi. Nous avons démontré, d'une part, que la production primaire planctonique du lac est relativement élevée et se situe dans une gamme moyenne pour les lacs africains (Mukankomeje et al. 1993). D'autre part, l'analyse du régime alimentaire d' $O$. niloticus montre que le phytoplancton est bien consommé par cette espèce, sans sélectivité marquée, excepté pour les Cyanobactéries filamenteuses, et que la ration journalière ingérée est du même ordre de grandeur que dans d'autres lacs africains eutrophes. Il faut donc rechercher les causes de la chute de production piscicole ailleurs, vraisemblablement dans la surexploitation des tilapias. Cette surexploitation est le résultat de l'absence d'une législation de pêche adéquate, de l'augmentation de la pression démographique à partir des années 1960, ainsi que de l'absence d'autres espèces à grande valeur commerciale, après la régression de $T$. rendalli. En effet, ce dernier tend à disparaître au Lac Muhazi faute de nourriture, les macrophytes immergés abondants dans les années 50 (Damas 1953) ayant pratiquement disparu. L'origine de cette régression est attribuable, d'une part, à une consommation par $T$. rendalli et, d'autre part, à une limitation par la lumière. On sait en effet que la transparence de l'eau s'est réduite d'un facteur 2 depuis les années 50, la profondeur actuelle de la zone photique étant d'environ $2 \mathrm{~m}$.

On peut émettre l'hypothèse que la surexploitation d' $O$. niloticus a entraîné la réduction de sa prédation sur les algues planctoniques de grande taille et a permis la prolifération de celles-ci. D'autre part, ce phytoplancton de grande taille, dominé par $M$. aeruginosa et $C$. hirundinella, est non ou peu consommé par le zooplancton. Il en résulte qu'une faible proportion de la production primaire du lac est exploitée directement dans la chaîne trophique.

Cette conclusion n'implique pas forcément que l'efficience trophique du lac soit réduite, dans la mesure où les autres voies trophiques (notamment via le zoobenthos et la production bactérienne) 
n'ont guère été explorées. Il faut en effet souligner que la biomasse piscicole du lac est largement dominée par des Haplochromis (162 kg.ha-1) au régime alimentaire varié et que d'autres espèces principalement benthophages comme des Clarias ont été introduites. Un meilleur rendement des pêches, tendant vers la production piscicole exploitable estimée à $65 \mathrm{~kg} \cdot \mathrm{ha}^{-1} \cdot \mathrm{an}^{-1}$, pourrait être atteint grâce à une exploitation des Haplochromis (Plisnier 1990), conjuguée à une diminution de la pression de pêche exercée sur $O$. niloticus et à un aménagement approprié du lac. Cet aménagement pourrait consister à restaurer la population d'O. niloticus par une protection de ses frayères, une réglementation de ses captures et par une réintroduction d'individus de souche pure. De telles mesures pourraient être un premier pas vers une réhabilitation écologique du lac et de ses ressources piscicoles.

\section{Remerciements}

Ce travail a été réalisé grâce au soutien financier, sous forme de bourses, de l'AGCD (Administration Générale de la Coopération au Développement, Belgique). De plus, il a été possible grâce au projet EAPLM (Etude et Aménagement Piscicole du Lac Muhazi), au sein duquel nous avons réalisé tous nos prélèvements, et enfin, grâce à l'encadrement et au soutien logistique de l'Unité d'Ecologie des Eaux Douces (Facultés Universitaires Notre-Dame de la Paix, Namur, Belgique).

\section{Travaux cités}

Abel R. 1973. - The trophic ecology of Cottus gobio (L.). PhD. Thesis, Oxford : $215 \mathrm{p}$.

Al-Hussaini A.H. \& Kholy A.A. 1954. - On the functional morphology of alimentary tract of some omnivorus teleost fish. Ibrahim University. Abbasiah (Cairo) and Institute of Oceanography-Al-Ghardaga : 17-39.

Beveridge M.C.M., Begum M., Frerichs G.N. \& Millar S. 1989. - The ingestion of bacteria in suspension by the tilapia Oreochromis niloticus. Aquaculture, $81: 373-378$.

Blache J. 1964. - Les poissons du lac Tchad et du bassin adjacent du Mayo Kebbi. Mémoire, ORSTOM : 239-241.

Bourrelly P. 1966. - Les algues d'eau douce : les algues vertes. Editions N. Boubée et Cie. Paris : 569 p.

Bourrelly P. 1968. - Les algues d'eau douce : les algues jaunes et brunes. Editions N. Boubée et Cie, Paris : 438 p.

Bourrelly P. 1970. - Les algues d'eau douce : Eugléniens, Péridiniens, algues rouges et algues bleues. Editions N. Boubée et Cie, Paris : $569 \mathrm{p}$.

Bunep 1988. - Etude pour le développement de la pêche et l'empoisonnement des lacs du Nord et de l'Est du Rwanda. Rapport définitif. Phase II. Ministère de l'Agriculture et de l'Elevage (Rwanda) : $434 \mathrm{p}$.

Compère P. 1974. - Algues de la région du lac Tchad. II. Cyanophycées. Cah. ORSTOM, sér. Hydrobiol., VIII : 165-198.
Compère P. 1975a. - Algues de la région du lac Tchad. III. Rhodophycées, Euglénophycées, Dinophycées, Chrysophycées, Xanthophycées. Cah. ORSTOM, sér. Hydrobiol,; IX (3): 167-192.

Compère P. 1975b. - Algues de la région du lac Tchad. IV. Diatomophycées. Cah. ORSTOM, sér. Hydrobiol., IX (4) : 203-290.

Compère P. 1976a. - Algues de la région du lac Tchad. V. Chlorophytes : 1ere Partie. Cah. ORSTOM, sér. Hydrobiol., $\mathrm{X}$ (2) : 77-118.

Compère P. 1976b. - Algues de la région du lac Tchad. VI. Chlorophytes : 2 Partie : Ulotrichophycées, Zygnématacées (1). Cah. ORSTOM, sér. Hydrobiol., X (3) : 135-164.

Compère P. 1977. - Algues de la région du lac Tchad. VII. Chlorophytes : 3e Partie: Desmidiées. Cah̆. ORSTOM, sér. Hydrobiol., XI (2) : 77-177.

Damas H. 1953. - Les lacs du Rwanda et leurs problèmes. Soc. Roy. Zool. de Belgique, Annales LXXXIV : 17-38.

De Vos L., Snoeks J. \& Thys Van Den Audenarde D. 1990. The effects of Tilapia introductions in lake Ruhondo, Rwanda. Env. Biology of Fishes, 27 : 303-308.

Dewan S. \& Shaha S.N. 1979. - Food and feeding habits of $T$. nilotica (diel and seasonal patterns of feeding). Bangladesh, J. Zoology, 7 (2) : 75-80.

Downing J.A., Plante C. \& Lalonde S. 1990, - Fish production correlated with primary productivity, not the morphoedaphic index. Can. J. Fish. Aquat. Sci., $47(10)$ : 1529-1536.

Fish G.R. 1955. - Food of Tilapia in East Africa. East African Fisheries Research Organization (EAFRO), Uganda : 58-89.

Fryer G. \& Iles T.D. 1972. - The Cichlid fishes of the great lakes of Africa. Oliver \& Boyd. Edinburgh : $641 \mathrm{p}$.

Ganf G.G. 1972. - The regulation of net production in lake George, East Africa. In Kajack Z. \& Hillbricht-Ilkowska A. (Eds). Productivity Problems of Fréshiwaters, Warsaw, Polish Scientific Publishers : 693-708.

Getachew T. 1989. - Stomach pH, feeding rythm and ingestion rate in Oreochromis niloticus L. (Pisces : Cichlidae) in Lake Awasa, Ethiopia. Hydrobiologia, 174 : 43-48.

Getachew T. \& Fernando C.H. 1989. - The food habits of an herbivorus fish (Oreochromis niloticus L.) in lake Awasa, Ethiopia. Hydrobiologia, 174 : 195-200.

Iltis A. 1980. - Les algues. In J.R. Durand \& Levêque C. (Eds). Flore et faune aquatiques de l'A frique Sahélo-Soudanaise. Editeurs Scientifiques Hydrobiologistes, ORSTOM, Tome I, Paris : 9-61.

Ivlev V.S. 1961. - Experimental ecology of feeding mechanism of the cichlid fish Tilapia esculenta Graham. Nature, 172 : 207-208.

Jarre A., Palomarès M.L., Soriano M.L., Sambilay C. \& Pauly D. 1991. - Some new analytical and comparative methods for estimating the food consumption of fish. ICES mar. Sci. Symp., 193 : 99-108.

Komarek J. \& Fott B. 1983. - Chlorophyceae (Grünalgen). Ordnug Chlorococcales. In Huber-Pestalozzi G., Das Phytoplankton des Süsswassers, Stuttgart : 1044 p.

Lauzanne L. 1976. - Régimes alimentaires et relations trophiques des poissons du lac Tchad. Cah. ORSTOM, sér. Hydrobiol., 9 (1) : 3-7.

Lebart L., Morineau A. \& Fénelon J.P. 1979. - Traitement des données statistiques. Méthodes et programmes. Dunod, Paris : 510 p. 
Liang Y., Melack J. \& Wang J. 1981. - Primary production and fish yieds in Chinese Ponds and lakes. Trans. Am. Fish. Society, $110: 346-350$.

Lind O.T. 1979. - Handbook of common methods in limno$\log y$ (second edition). London : $199 \mathrm{p}$.

Lowe-McConnell R.H. 1958. - Observations on the biology of Tilapia nilotica Linné in East African waters (Pisces : Cichlidae). Rev. Zool. Bot. Afr., LVII, 129-170.

McConnell M.J., Lewis S. \& Olson J.E. 1977. - Gross photosynthesis as an estimator of potential fish production. Trans. Am. Fish. Soc., $106:$ : 417-423.

Melack J.M. 1976. - Primary productivity and fish yields in tropical lakes. Trans. Am. Fish. Soc., 105 (5) : 575-580.

Micha J.C. \& Frank V. 1992. - Ressources et caractéristiques piscicoles des lacs rwandais (bassin nilotique). Bull. Séanc. Acad. r. Sci. Outre-Mer 37 (3) : 379-398.

Moriarty D.J. 1973. - The physiology of digestion of blue-green algae in the cichlid, Tilapia nilotica. International Biological Programme, RSAFBT, Lake George, Uganda, J. Zool., $171: 15-23$.

Moriarty D.J. \& Moriarty C.M. 1973. - The assimilation of carbon from phytoplankton by two herbivorous fishes : Tilapia nilotica and Haplochromis nigripinnis. J. Zool., 171 : 41-45.

Mukankomeje R. 1992. - Production algale et consommation par le tilapia, Oreochromis niloticus L., au Lac Muhazi (Rwanda). Thèse de Doctorat, FUNDP, Namur, Belgique : $254 \mathrm{p}$.

Mukankomeje R, Plisnier P.D., Descy J.P. \& Massaut L. 1993. - Lake Muhazi, Rwanda : limnological features and phytoplankton production. Hydrobiologia, $257: 107-120$.

Naca 1989. - Integrated fish farming in China. NACA Technical Manual 7. A Word Food Day 1989. Publication of the Network of Aquaculture Centers in Asia and the Pacific. Bangkok, Thailand, $278 \mathrm{p}$.

Northcott M.E. \& Beveridge M.C.M. 1988. - The development and structure of pharyngeal apparatus associated with filter feeding in tilapias (Oreochromis niloticus). J. Zool., 215 : 133-149.

Northcott M.E. \& Beveridge M.C.M. \& Ross L.G. 1991. - A laboratory investigation of filtration and ingestion rates of tilapia, Oreochromis niloticus, feeding on two species of bluegreen algae. Environ. Biol. of Fishes, 31 : 75-85.

Oglesby T.R. 1977. - Relationships of fish yield to lake phytoplankton standing crop, production and morphoedaphic factors. J. Fish. Res. Board Can., 34 : 2271-2279.

Olah J., Sinha V.R.P., Ayyappan S., Purushothanc C. \& Radheyshyams S. 1986. - Primary Production and Fish Yields in Fish Ponds under different Management Practices. Aquaculture, $58: 111-122$.

Palomarès M.L.D. 1991. - La consommation de nourriture chez les poissons : Etude comparative, mise au point d'un modèle prédictif et application à l'étude des réseaux trophiques. Thèse de Doctorat, Inst. Nat. Polyt. Toulouse, France : 210 p.
Palomarès M.L.D. \& Pauly D. 1989. - A multiple regression model for predicting the food consumption of marine fish populations. Aust. J. Mar. Freshwater Res., 40 : 259-273.

Palomarès M.L.D. \& Pauly D. 1991. - Models for estimating the food consumption of Tilapias. In The Third International Conference on Tilapia in Aquaculture (ISTA III), Abidjan Côte d'Ivoire : ICLARM Contribution, $\mathrm{n}^{\circ} 776: 18 \mathrm{p}$.

Pauly D. 1980. - On the interrelationships between natural mortality, growth parameters and mean environmental temperature in 175 fish stocks. J. Cons. Int. Explor. Mer, 39 (2) : 175-192.

Pauly D. 1986. - A simple method for the estimating the food consumption of the fish populations from growth data food conversion experiments. Fish. Bull., 84 (4) : 827-840.

Plisnier P.D. 1989. - Etude hydrobiologique et développement de la pêche au lac Muhazi (bassin de l'Akagera, Rwanda). ACDST (ULg), UNECED (FNDP), MINAGRI-AGCD, rapport final (1986-1988) : $178 \mathrm{p}$.

Plisnier P.D. 1990. - Ecologie comparée et exploitation rationnelle de deux populations de Haplochromis spp. (Teleostei, Cichlidae) des lacs Ihema et Muhazi (Rwanda). Thèse de Doctorat, UCL Louvain-la-Neuve, Belgium : $328 \mathrm{p}$.

Ryder R.A. 1965. - A method for estimating the potential fish production of North temperate lakes. Trans. Am. Fish. Soc., 94 : 214-218.

Sirven P., Gotanègre J.F. \& Prioul C. 1974. - Géographie du $R$ wanda. Ed. A. De Boeck, Bruxelles, Ed. rwandaises, Kigali : $174 \mathrm{p}$.

Smith E.V. \& Swingleh S. 1938. - The relationship between plankton production and fish production in ponds. Trans. Am. Fish. Soc., 68 : 309-315.

Sournia A. 1978. - Phytoplankton manual. Musée National d'Histoire Naturelle. Paris (UNESCO) : $282 \mathrm{p}$.

Sreenivisan A. 1972. - Energy transformation through primary productivity and fish production in some tropical freshwater impoundments and ponds. In Kajack Z. \& HillbrichtIlkowska A. (Eds) Productivity Problems of Freshwaters, Warsaw, Polish Scientific Publishers : 505-514.

Trewavas E. 1983. - Tilapiine fishes of the genera Sarotherodon, Oreochromis and Danikilia. British Museum Nat. Hist. : $583 \mathrm{p}$.

Tudorancea C., Fernando C.H. \& Paggi J.C. 1988. - Food and feeding ecology of Oreochromis niloticus juveniles in lake Awasa (Ethiopia). Arch. Hydrobiol. suppl., 79 : 267-289.

Ufodike E.B.C. \& Wada R.K. 1991. - Feeding habits of tilapia, Sarotherodon nilotica (Perciformis : Cichlidae) fry in Jos, Nigeria. Rev. Biol. Trop., 39 (2) : 189-192.

Ugwamba A.A.A. \& Adebis A. 1992. - The food and feeding ecology of Sarotherodon melanotheron (Ruppell) in a small freshwater reservoir in Ibadan, Nigeria. Arch. Hydrobiol., 124 (3) : 367-382.

Vollenweider R.A. (Ed.) 1974. - A manual on methods for measuring primary production in aquatic environments. IBP Handbook 12, Blackwell Scientific Publications, Oxford, 225 p. 